\title{
HERRAMIENTA WEB DE DIAGNÓSTICO PARA COVID-19 MEDIANTE EL ANÁLISIS DE RADIOGRAFÍAS CON IA: PROYECTO X-COV
}

\author{
Rocío Silleras-Aguilar, Adrián Belarra Rodríguez, Paula del Burgo Corella, Nerea Encina Baranda, \\ Clara Freijo Escudero, Isabel Gallego Llorente, Pablo Galve, Iñaki García del Pozo, \\ Alejandro López-Montes, Olivia M. Giza, Ober Van Gómez López y Joaquín L. Herraiz \\ Grupo de Física Nuclear / IPARCOS. Universidad Complutense de Madrid \\ Facultad de Ciencias Físicas. Plaza de las Ciencias 1, Madrid, 28040, España
}

\begin{abstract}
RESUMEN
El proyecto X-COV está desarrollando una herramienta web de análisis de radiografías basado en inteligencia artificial para la detección y evaluación de pacientes de COVID-19. En la creación del modelo basado en Deep Learning se han utilizado las herramientas de aprendizaje automático tales como TensorFlow, Keras y Tensorflow.js para su implementación en una página web. El modelo ha sido entrenado con miles de radiografías en formato DICOM y datos de repositorios públicos y de hospitales colaboradores. Este tipo de proyecto demuestra la capacidad que tienen las herramientas web para ayudar en situaciones complejas a los médicos.
\end{abstract}

\section{PALABRAS CLAVE}

Inteligencia Artificial, Deep Learning, Covid-19, Radiografías, TensorFlow, Keras

\section{INTRODUCCIÓN}

La actual crisis sanitaria global ha impulsado la investigación y desarrollo de numerosos proyectos de salud basados en inteligencia artificial (IA) situándose como una herramienta de ayuda de gran potencial. En este trabajo se presenta la herramienta web del Proyecto X-COV que permite el acceso a un modelo entrenado de IA que analiza radiografías de tórax (CXR, chest $x$-ray) para detectar casos COVID-19 y evaluar la progresión de la enfermedad aportando a los radiólogos información de interés para facilitar y agilizar su labor.

La IA ya ha sido utilizada con anterioridad en el análisis de radiografías y en la detección neumonías bacterianas y víricas anteriores al COVID-19. Lo más interesante de la IA es su versatilidad, debido a que modelos pensados para unas enfermedades o dolencias sirven de base y pueden adaptarse a otras. Las primeras aplicaciones de la IA en esta pandemia comenzaron así. La gran inversión pública y privada ha fomentado el rápido desarrollo de este tipo de proyectos. Para tener una visión amplia de las propuestas y trabajos previos relacionados se recomienda el artículo de Shi et al. (2020), así como el apartado de recursos de la web de X-COV (2020) donde se han recopilado tanto las referencias como los conjuntos de datos públicos usados.

En este trabajo mostramos cómo se ha implementado este proyecto, sus aplicaciones y líneas futuras. 


\section{DESCRIPCIÓN DEL PROYECTO Y SU DESARROLLO}

\subsection{Interés del Análisis de CXR con IA y Descripción General del Proyecto}

Las diversas técnicas de imagen médica están siendo muy valiosas a la hora de constatar y evaluar el estado de los pacientes con síntomas compatibles o comprobar falsos negativos en las PCR (Polymerase Chain Reaction). Tal es su uso, que la Organización Mundial de la Salud ha creado una guía específica para el COVID-19 (WHO, 2020). Las CXR son, en muchos casos, la mejor o la única opción al ser más comunes, accesibles, baratas, tener un menor tiempo de ejecución y por los equipos de radiografía portátiles, que permiten realizar pruebas sin trasladar al enfermo, con los beneficios que esto conlleva, tanto para el paciente como para el centro.

Atendiendo al entorno donde nació este proyecto, según el protocolo de manejo clínico español, las CXR han sido fundamentales para el manejo de pacientes, evaluar su estado y el progreso de la enfermedad. Se han usado como parte de la toma de decisiones para ingresos hospitalarios, evaluar el progreso de la enfermedad y al valorar si un paciente ingresado estaba lo suficientemente recuperado para recibir el alta. La IA puede ser muy útil en estas tareas, sobre todo en los casos en los que el análisis de CXR no es concluyente, ante falsos negativos de las CXR o los PCR, o ante una saturación de los centros. Aquí la IA puede marcar la diferencia al dar automáticamente una evaluación objetiva de la imagen, marcando sus zonas de interés y aportando una clara métrica, permitiendo una valoración y toma de decisiones médicas más ágil y mejor informada.

$\mathrm{X}-\mathrm{COV}$ es un proyecto de investigación del Grupo de Física Nuclear de la Universidad Complutense de Madrid cuyo objetivo es poner a disposición de los radiólogos este tipo de herramientas de análisis automático de CXR con IA. El modelo fue entrenado en primer lugar con open data disponible en repositorios públicos de CXR. Todos son señalados en la base de datos open de X-COV (2020). Tras la primera aproximación se han ido añadiendo imágenes y datos de los Hospitales que colaboran con el proyecto para mejorar sus resultados.

Desde un inicio se plantearon dos líneas de actuación. Por un lado, crear la herramienta online, abierta y de libre acceso con fines de investigación (no certificada para su uso clínico) que aquí se describe. Esta versión permite su acceso a médicos e investigadores de otros países, sirviendo además de plataforma de pruebas para las nuevas características que se van implementando en las redes. A la vez facilita la difusión y el interés por el proyecto en su conjunto. La segunda línea es el desarrollo de una versión para su uso clínico. Requiere certificación con marcado CE (Conformité Européenne) y/o el de FDA (Food and Drug Administration) de dispositivos médicos y estará adaptada para ser integrada en el sistema PACS (Picture Archiving and Communication System) de los hospitales y con algunas máquinas de radiología de proveedores específicos.

\subsection{Desarrollo de la Aplicación Web}

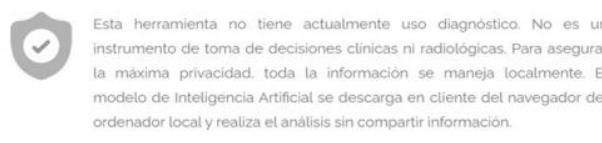

CHEST X-RAY ANALYZER - COVID-19
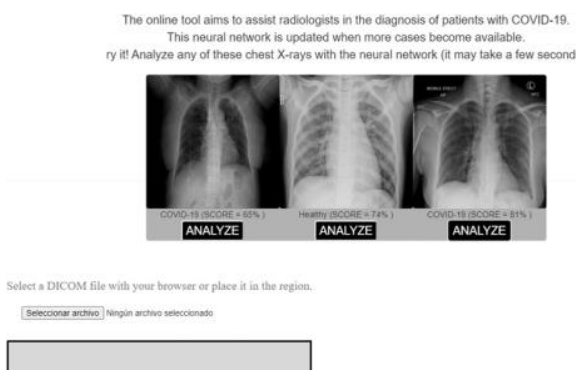

COVID-19: CHEST X-RAY ANALYZER
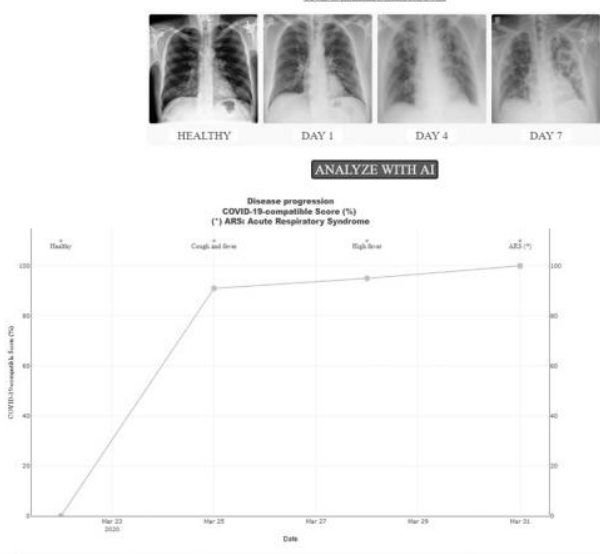

Figura 1. Elementos de la herramienta de análisis de CXR y la de evaluación por días de la web de X-COV 
El modelo y la aplicación web son accesibles a través de la web de X-COV (2020) donde se especifica su tipo de uso y el manejo local de la información. Allí también se encuentra la base de datos open del proyecto cuyo fin es fomentar la investigación y facilitar el desarrollo de otros proyectos relacionados con el diagnóstico de COVID-19 mediante CXR.

El modelo usa técnicas de Deep Learning (aprendizaje profundo), aportando en cada caso un análisis inmediato con una puntuación sobre el nivel de gravedad del paciente y si es neumonía tipo COVID-19, permitiendo un triage más fiable. Ese valor sirve además como referencia adicional al evaluar la evolución del paciente. La herramienta también permite combinar la información radiológica con otra información de interés como edad, género, días desde el inicio de síntomas, temperatura o alguna otra indicación relevante.

La herramienta trabaja con imágenes de entrada en formato DICOM (Digital Imaging and Communication In Medicine) (DICOM 2020), el estándar para este tipo de imágenes médicas, y con formatos gráficos comunes como JPG y PNG. El análisis de las imágenes se realiza en la web del dispositivo, permitiendo usarlas sin enviarse a ningún servidor, permaneciendo así de forma local y asegurando la privacidad de los datos.

El modelo está creado con las herramientas de aprendizaje automático Keras (s.f.), TensorFlow (s.f.), y Tensorflow.js para su implementación web. A pesar de que el esquema web impone ciertas limitaciones en la complejidad de la red que se puede llegar a usar, se optó por él al facilitar su accesibilidad, manejo y escalabilidad. Al no necesitar hacer cálculos en el servidor permite un gran número de accesos simultáneos.

Para su entrenamiento, en primer lugar, se utilizaron cientos de CXR de publicaciones y repositorios públicos relacionados con COVID-19 y con otras afecciones pulmonares. Estos datos se pueden encontrar en la base de datos open de la web de X-COV (2020). Esta primera aproximación tuvo una precisión del 91,7\% y evitó los falsos negativos en un $100 \%$ obteniendo resultados muy interesantes.

El siguiente paso fue pre-procesar, clasificar y estandarizar los nuevos datos y CXR de pacientes con diagnóstico confirmado en COVID-19 provenientes de los hospitales que colaboran con el proyecto, usando primero los del Hospital Clínico San Carlos de Madrid gracias a su Servicio de Radiología. Con la optimización de las redes y su reentrenamiento, con estos nuevos y mejores datos, han ido perfeccionando continuamente la herramienta. Este proceso se continuará con los datos e imágenes del resto de colaboradores, extendiendo esta actividad durante todo el proyecto. Ahora se encuentra en una nueva iteración que será finalizada en octubre-noviembre, momento en el que se hará pública junto a sus resultados en la web de X-COV (2020). Para asegurar la reproducibilidad de los resultados y mantener su consistencia en el tiempo, cuando se actualice el modelo de IA se ofrecerá la opción de seguir usando versiones anteriores.

\subsection{Desarrollo del Servidor Web Local}

Los hospitales que quieran utilizar esta herramienta podrán usar una versión basada en un servidor web local, para que sea más fácil acceder desde el sistema PACS del hospital. Igual que en el caso anterior, el software analizará la información suministrada y devolverá una valoración con el nivel de gravedad del paciente y una breve indicación de las características más importantes que el modelo utilizó para realizar dicha valoración.

El análisis de imágenes se realizará en la propia web del dispositivo, de forma similar a otras herramientas como Chester (MLMED y Mila Medical, 2019). Esto permite usar imágenes sin tener que ser anonimizadas, al permanecer en todo momento localmente y no ser enviadas al exterior, favoreciendo la seguridad. En cualquier caso y dado el interés de esa opción, también se contempla la posibilidad de guardar las imágenes e información de cada caso correctamente anonimizadas en el servidor, para permitir así el seguimiento de pacientes que reciben varias radiografías durante su tratamiento.

Hasta obtener su certificación, se indicará que no se trata de un producto sanitario certificado con las restricciones de uso que esto implica y la obligación de aceptar esas condiciones antes de usar la herramienta.

La plataforma almacenará los datos de forma segura, cumpliendo todas las medidas de seguridad indicados en los reglamentos españoles y europeos. En todo momento, en la aplicación web y en el servidor web local el manejo de imágenes y datos se hará respetando los derechos de los pacientes y la legislación vigente. Esto es, la Ley Orgánica 3/2018 (BOE) y el Reglamento UE 2016/679 (Parlamento Europeo, 2016).

Nuevamente, para asegurar la reproducibilidad de los resultados y mantener su consistencia en el tiempo, si se actualiza el modelo de IA se advertirá de ello y se permitirá seguir usando versiones anteriores. 


\subsection{Mantenimiento y Mejora Continuada de la Herramienta y del Proyecto}

El modelo se está mejorando a través de la sucesiva optimización y reentrenamiento de sus redes neuronales. Para perfeccionar la herramienta y sus resultados, durante todo el proyecto se cuenta con el apoyo de radiólogos y médicos de otras especialidades, dado que el feedback que aportan permite atender a las opiniones del usuario final de una forma ágil, adaptando rápidamente la herramienta a sus requerimientos y necesidades. También se ha ofrecido la herramienta y se mantiene contacto con investigadores y radiólogos de otros países, poniendo especial atención en aquellos con rentas más bajas. Profesionales de Brasil, Colombia, Venezuela y Costa Rica ya se han mostrado muy interesados por las herramientas. Ellos pueden aportar una perspectiva diferente, obteniendo así una visión más global.

El proyecto ya se encuentra colaborando con otros grupos de investigación que desarrollan proyectos relacionados a nivel nacional y se continúan buscando nuevas colaboraciones con otros grupos a nivel mundial que trabajen en estas mismas líneas, para compartir datos, analizar y evaluar modelos, y crear otras relaciones y sinergias de interés que fortalezcan aún más al proyecto.

El Proyecto X-COV ha recibido varios reconocimientos a nivel nacional e internacional. Resultó ganador absoluto del hackathon internacional \#CodeTheCurve de la UNESCO, IBM y SAP, finalistas del hackathon nacional \#VenceAlVirus organizado por la Comunidad de Madrid y el ganador del programa de acompañamiento de CompluEmprende de la Universidad Complutense. Como premio en estas convocatorias, se ha recibido un importante apoyo de formación y asesoramiento, mentorías de expertos, financiación para recursos y difusión. Por último, queda mencionar el interés que el proyecto ha generado en diversas empresas tecnológicas, destacando las importantes financiaciones en recursos informáticos que ha recibido por parte de Google Cloud y Amazon AWS, y el uso del clúster supercomputación de SENAI CIMATEC en Brasil. Todas estas colaboraciones, ayudas y recursos fomentan una evolución rápida y fluida, facilitando la mejora continua de todo el proyecto y sus herramientas.

\section{CONCLUSIÓN}

El proyecto X-COV muestra cómo es posible facilitar el acceso, a través de una página web, a un modelo de inteligencia artificial que permita la evaluación de pacientes de COVID-19 a partir de sus radiografías de tórax e información básica del paciente. En este proyecto se ha prestado una especial atención a los requerimientos del entorno clínico, como el uso del formato DICOM y un contacto activo con hospitales y usuarios para atender las necesidades de ambos. Los resultados son prometedores, pero se realizará una mejora continuada con el acceso a nuevos casos.

\section{AGRADECIMIENTOS}

X-COV agradece a los diferentes hospitales, instituciones y empresas que están apoyando y colaborando con el proyecto: Hospital Clínico San Carlos de Madrid, Hospital Universitario de Fuenlabrada, Hospital Universitario 12 de Octubre, Hospital Rúber Juan Bravo, Universidad Complutense de Madrid, Programa CompluEmprende, Hackathon Madrid \#VenceAlVirus, Consejería de Ciencia, Universidades e Innovación de la Comunidad de Madrid, Madri+D, CSIC, DASEL, Hackathon UNESCO \#CodeTheCurve, SAP, IBM, Google Cloud, Amazon AWS y Repsol-Sinopec.

\section{REFERENCIAS}

BOE (Boletín Oficial del Estado) [WWW Document], 2018. BOE.es - Documento BOE-A-2018-16673 URL https://www.boe.es/eli/es/lo/2018/12/05/3 (accessed 8.10.20).

DICOM [WWW Document], 2020. URL http://dicom.nema.org/medical/dicom/current/output/html/part10.html (accessed 8.1.20).

Keras: the Python deep learning API [WWW Document], s.f.. Keras. URL https://keras.io/ (accessed: 30.9.20). 
MLMED y Mila Medical [WWW Document], 2019. Chester the AI Radiology Assistant (V3). URL https://mlmed.org/tools/xray/ (accessed 8.10.20).

Parlamento Europeo, 2016. Reglamento (UE) 2016/679 del Parlamento Europeo y del Consejo, de 27 de abril de 2016 (Reglamento general de protección de datos). Diario Oficial de la Unión Europea.

Shi, F., Wang, J., Shi, J., Wu, Z., Wang, Q., Tang, Z., He, K., Shi, Y., Shen, D., 2020. Review of Artificial Intelligence Techniques in Imaging Data Acquisition, Segmentation and Diagnosis for COVID-19. IEEE Rev. Biomed. Eng. 1-1.

TensorFlow [WWW Document], s.f.. TensorFlow. URL https://www.tensorflow.org/?hl=es-419 (accessed: 30.9.20).

World Health Organization (WHO), 2020. Use of chest imaging in COVID-19: a rapid advice guide. World Health Organization. https://apps.who.int/iris/handle/10665/332336

X-COV [WWW Document], 2020. Clasificación de pacientes con IA. URL http://tomografia.es (accessed 8.1.20). 